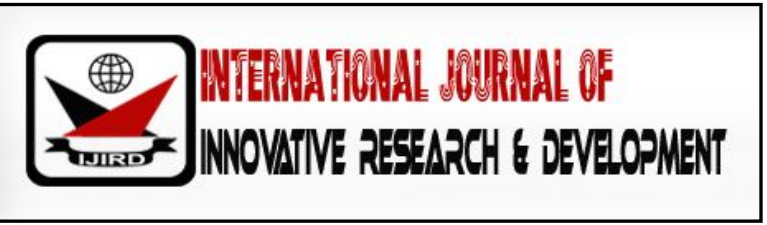

ISSN 2278 - 0211 (Online)

\title{
Quantification of Fracture of Rock Analogue Using a Fractal Geometry Tool - Box-Counting
}

\author{
Wilson, M. C. \\ Faculty of Civil-Geo, Department of Geological Engineering, KNUST, Kumasi, Ghana
}

\begin{abstract}
:
Quantification of flow patterns and also that of rock analogue using the concepts of fractal geometry are less researched and discussed, unlike quantification of the original rock. I thus came with the idea to quantify complex flow patterns of fractures from experiments of rock analogue and its significance to tectonic activities and magmatic rocks. Rock analogue is a structure that is similar in function to a rock but the origin or chemical composition is different. A series of experiments was organized to portray different forms of flow patterns of a rock analogue in accordance to different acts of viscosities. The specific rock analogue used in this experimental process was a fine-grained starch. The experiment reveals the fractal and fracture nature in flow patterns as different forms of weight \% and different acts of viscosities and densities exhibit different forms and sizes of fractures. Different weights of starch were sampled with a specific and constant weight of $100 \mathrm{~g}$ of water. The rock analogue experiment is fully based on the principles of fractal geometry. To quantify this flow experiment, two different methods of fractal geometry are applied, namely the MORFA and Box-counting. An average fractal dimension of 1.62 on cracks or faults was estimated which corresponds to the fractal dimensions of 1.4 - 1.7, which Barton (1995) conducted on the systematic studies on exposed joint and fault trace patterns on Yucca Mount in Nevada and also the model of fracture pattern proposed by Takayasu (1985).
\end{abstract}

Keywords: Flow patterns, rock analogue, viscosities, fractal geometry, fractal dimension

\section{Introduction}

The concept of fractals was introduced by Mandelbrot (1967) in a geological context. He noticed that the length of a rocky coastline increased as the length of the measuring rod decreased according to a power law, and thus he associated the power with a fractal dimension. Mandelbrot (1982) used fractal concepts to generate synthetic landscapes that looked remarkably similar to actual landscapes. The definition of a fractal distribution is that the number of objects $\mathrm{N}$ with a characteristic size greater than $r$ scales with the relation $\mathrm{N}(\mathrm{r}) \sim \mathrm{r}^{-\mathrm{D}} \cdot \mathrm{N}=\underline{\mathrm{C}} \mathrm{r}^{\mathrm{D}}$

The power determines the fractal dimension (of the coastline) and $\mathrm{C}$ a constant of proportionality. This paper presents an extensive evaluation of the methods to quantify the fractal dimension of fractures of a rock analogue (starch). Two methods: Mapping of Rock Fabric Anisotropy (MORFA) and Box-Counting are applied to 2-D flow experiment data extracted from the photographed data of the flow experiment of a mixture of water and starch. Quantification of fractures using MATLAB and other tool boxes have been commonly employed on rocks. I thus thought of quantifying this time an analogue of rock instead. Rock analogue is a structure that is similar in function to a rock but the origin or chemical composition is different. The main aim of this paper is to quantify complex flow patterns of fractures from experiments of rock analogue and its significance to tectonic activities and magmatic rocks. The specific aims of this paper are as follows: To determine the rate of flow of the different weight capacities of the rock analogue; analyse the flow rate of the rock analogue with respect to the flow of magmatic rocks; to determine the anisotropy of cracks or fractures of the rock analogue; and also, the use of MORFA and Box-Counting to quantify and attain the required results. 


\section{Materials and Methodology}

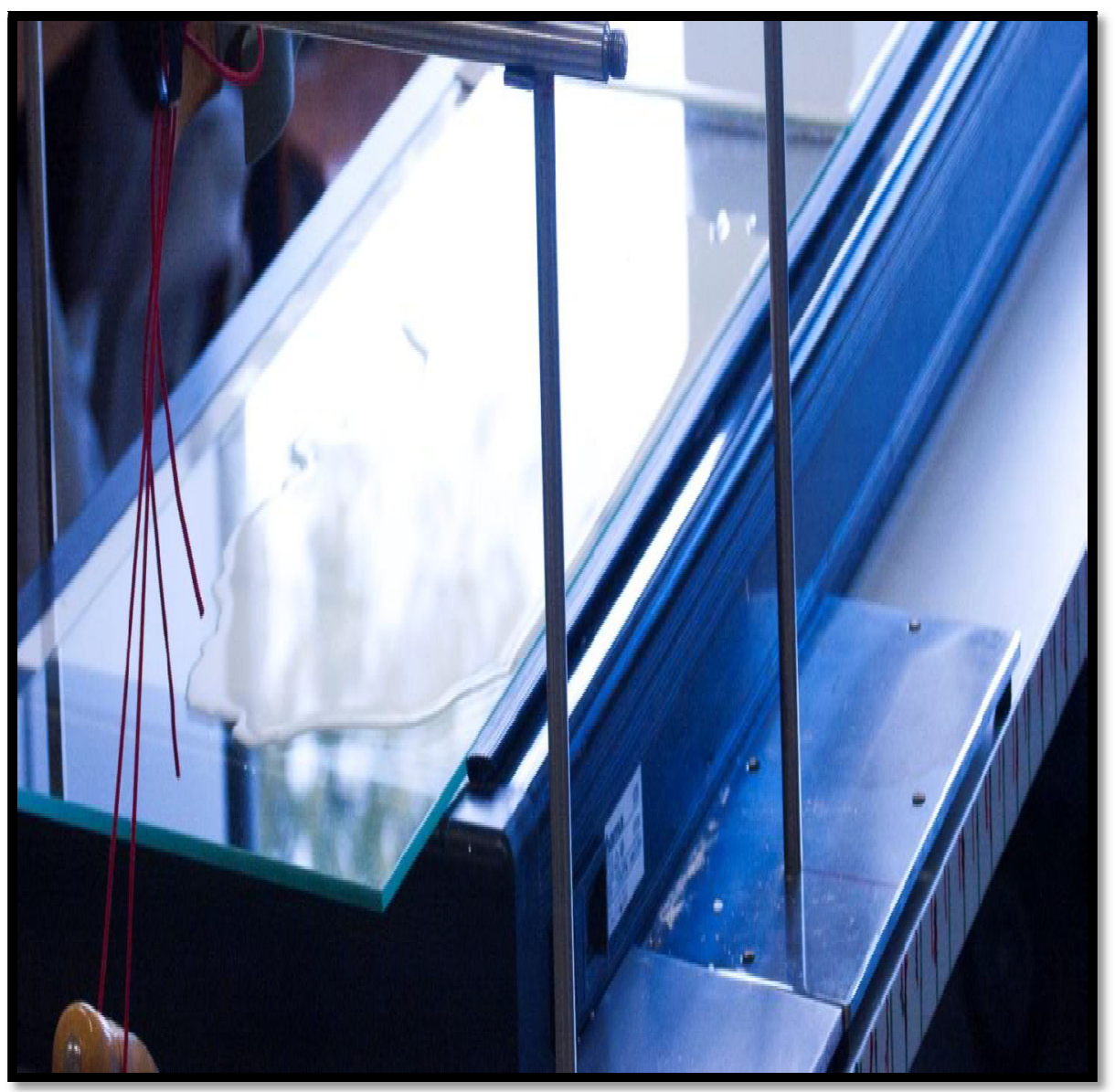

Figure 1: A Mixture of Starch and Water Flowing on a Glass Plate

The whole experiment is to reveal the rate of flow of a raw complex rock analogue and how to quantify it. The rock analogue chosen in this wise was starch ("feine Speisestärke in German shop, the starch used to mix with powdered plantain to make "fufu" in Germany). I used a glass cup to measure the water and starch contents in grams. The mixed starch and water content was placed on a stirring machine at room temperature. With the aid of a small plastic stirring substance, the mixture was homogeneously stirred. At the start of the stirring, a stop clock would be started to notify the time at which the content was homogeneously stirred. A glass plate was laid on a light glass box. A plastic bottle was used to hold and release the starch and water mixture to flow on the glass plate on the glass box to observe its rate of flow. Two metallic clamp stands on both sides of the glass box connected to one another was made to hold the plastic bottle and its contents (Figure 1). A geological compass was made to set the horizontal stand of the glass plate and glass box at zero level.

The different experiments were carried out under room temperature in a geology laboratory. A literal and constant weight of 100 grams of water was used throughout the experiments. However, different weight capacities of the rock analogue (starch) were used to mix with the water. Three different weight capacities of starch were measured at three different viscosity rates (low, medium and high viscosities). At low viscosity, $40 \mathrm{~g}$ of starch was measured, whilst $60 \mathrm{~g}$ and $80 \mathrm{~g}$ of starch were measured for medium and high viscosities respectively. Note that different forms of weight experiments were carried out before I could come out with this decision of $20 \mathrm{~g}$ intervals of different forms of viscosity. In performing the different forms of viscosity experiments, the low viscosity at $40 \mathrm{~g}$, the medium viscosity at $60 \mathrm{~g}$ and the high viscosity at $80 \mathrm{~g}$ of raw starch were weighed and added to the constant $100 \mathrm{~g}$ of water in a water cup containing a small plastic stirring substance. The contents were placed on the stirrer machine and allowed at different times to be homogeneously stirred. A stop clock was set immediately the starch and water contents were placed on the stirrer machine (figure 2). Different times of 5, 8 and 11 minutes were recorded for low, medium and high viscosities respectively. After the contents had been homogeneously stirred, the mixture would be poured into the plastic bottle which was being held by two metallic clamps and the contents allowed at a free space to flow along the glass plate laid on the glass box (Figure 1). The distance between the glass plate and the plastic bottle (with the contents) was measured at approximately three millimetres. These experiments were performed with the glass plate set at the zeroing level (the plate at a totally horizontal level). 


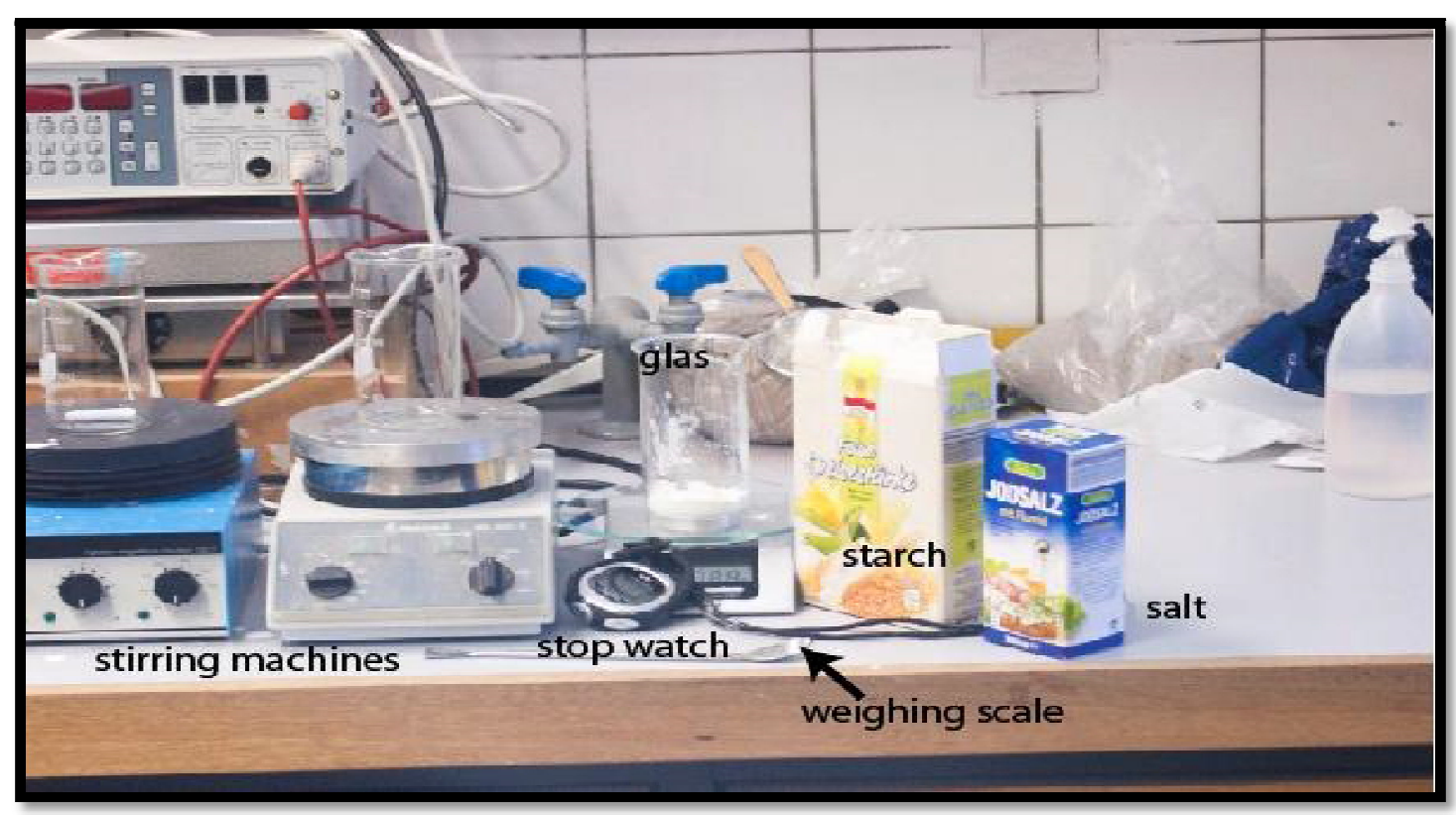

Figure 2: Apparatuses and Substances Used for the Experiment - Measuring Glass Cup,

Weighing Scale, Stirring Machine, Plastic Substance, Stop Watch and Starch

The flow experiments took two days to dry up. The Nixon 70 camera was implored to take photographs of all the dried raw starch and water mixtures. The photos were taken at overlaps of $2.5 \mathrm{~cm}$ in both the column and row directions in 3 columns times 5 rows giving fifteen photos. The distance from the vertical position of the camera to the measured starch was set at $12 \mathrm{~cm}$ and the camera aperture of F11 at a set time of 10 seconds. These parameters were set constant for all the experiments. This is because a slight change of any of these parameters and their resolutions would affect the quality of the pattern and such results in biasing of the outcome. For instance, colour contrast between two different photos at different positions of the same experiment or different experiments lead to inaccuracy of the images. These fifteen photos were being stitched together with the aid of both the PT Gui and Adobe Photoshop software and converted to grey-scale images in order to separate the starch and cracks of the rock analogue. To process the image begins with a clean-up using the Adobe Photoshop software (Peternell \& Kruhl, 2009) and this will convert the images into black and white images. The white phase of image represents cracks or fractures whilst the black phase of the output image represents the starch. The quality of the pattern or fabrics depends on the quality of the generated resolution of the image.

Laboratory data was mostly in the form of digital photography. The digitized images were converted to tiff format (table 1). Each of the fifteen small pictures of a particular image represents only a single fraction of the total structure (Figures $3,4,5)$.

\begin{tabular}{|c|c|c|c|c|}
\hline $\begin{array}{l}\text { Types of } \\
\text { Viscosity }\end{array}$ & $\begin{array}{c}\text { Weight of Water } \\
\text { (G) }\end{array}$ & $\begin{array}{c}\text { Weight of Starch } \\
\text { (G) }\end{array}$ & Raw Data & Tiff Image \\
\hline \multirow{14}{*}{$\begin{array}{c}\text { Low or Medium or } \\
\text { High }\end{array}$} & \multirow[t]{14}{*}{100} & \multirow[t]{14}{*}{40 or 60 or 80} & DSC 3996.NEF & DSC 3996.tif \\
\hline & & & DSC 3997.NEF & DSC 3997.tif \\
\hline & & & DSC 3998.NEF & DSC 3998.tif \\
\hline & & & DSC 3999.NEF & DSC 3999.tif \\
\hline & & & DSC 4000.NEF & DSC 4000.tif \\
\hline & & & DSC 4001.NEF & DSC 4001.tif \\
\hline & & & DSC 4002.NEF & DSC_4002.tif \\
\hline & & & DSC 4003.NEF & DSC 4003.tif \\
\hline & & & DSC 4 4004.NEF & DSC_4004.tif \\
\hline & & & DSC 4005.NEF & DSC 4005.tif \\
\hline & & & DSC 4006.NEF & DSC 4006.tif \\
\hline & & & DSC 4007.NEF & DSC 4007.tif \\
\hline & & & DSC 4008.NEF & DSC 4008.tif \\
\hline & & & DSC 4009.NEF & DSC 4009.tif \\
\hline
\end{tabular}

Table 1: Raw Data and Tiff Images towards Black and White Stitched Images of the Analogue 


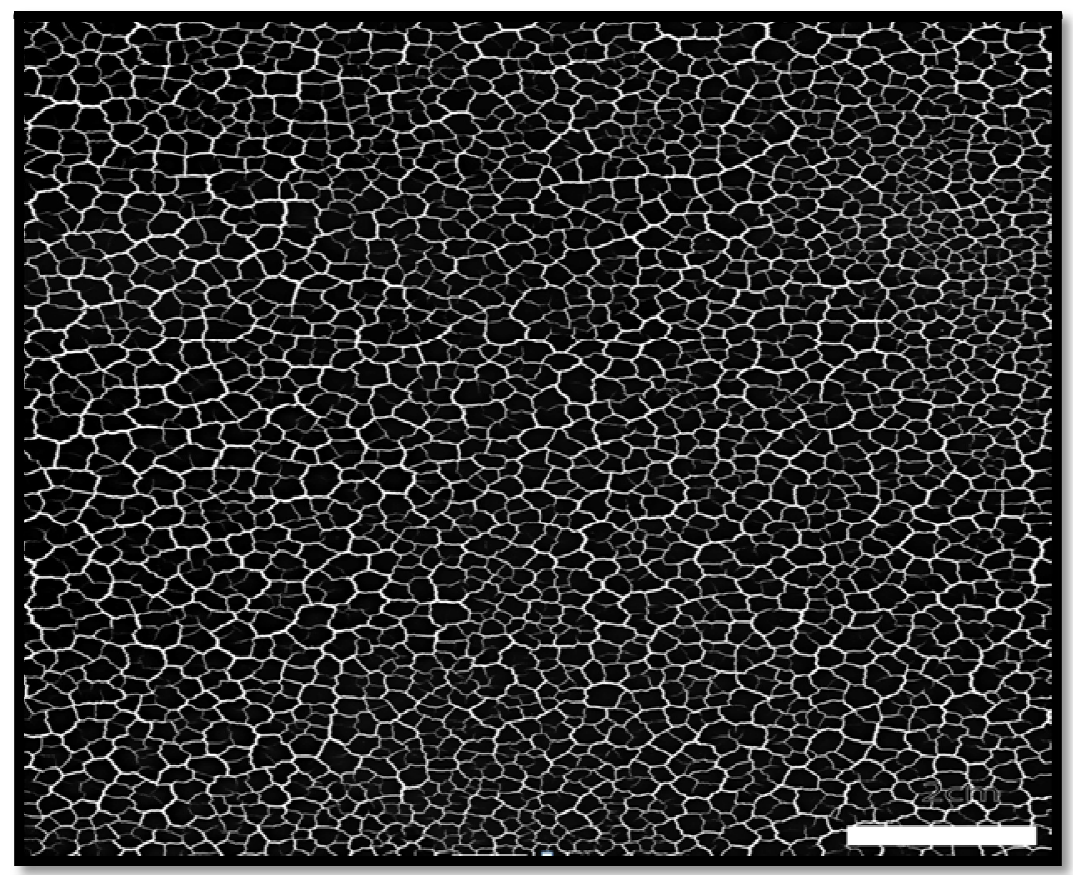

Figure 3: Low Viscosity Horizontal Raw Starch Experiment of 40g

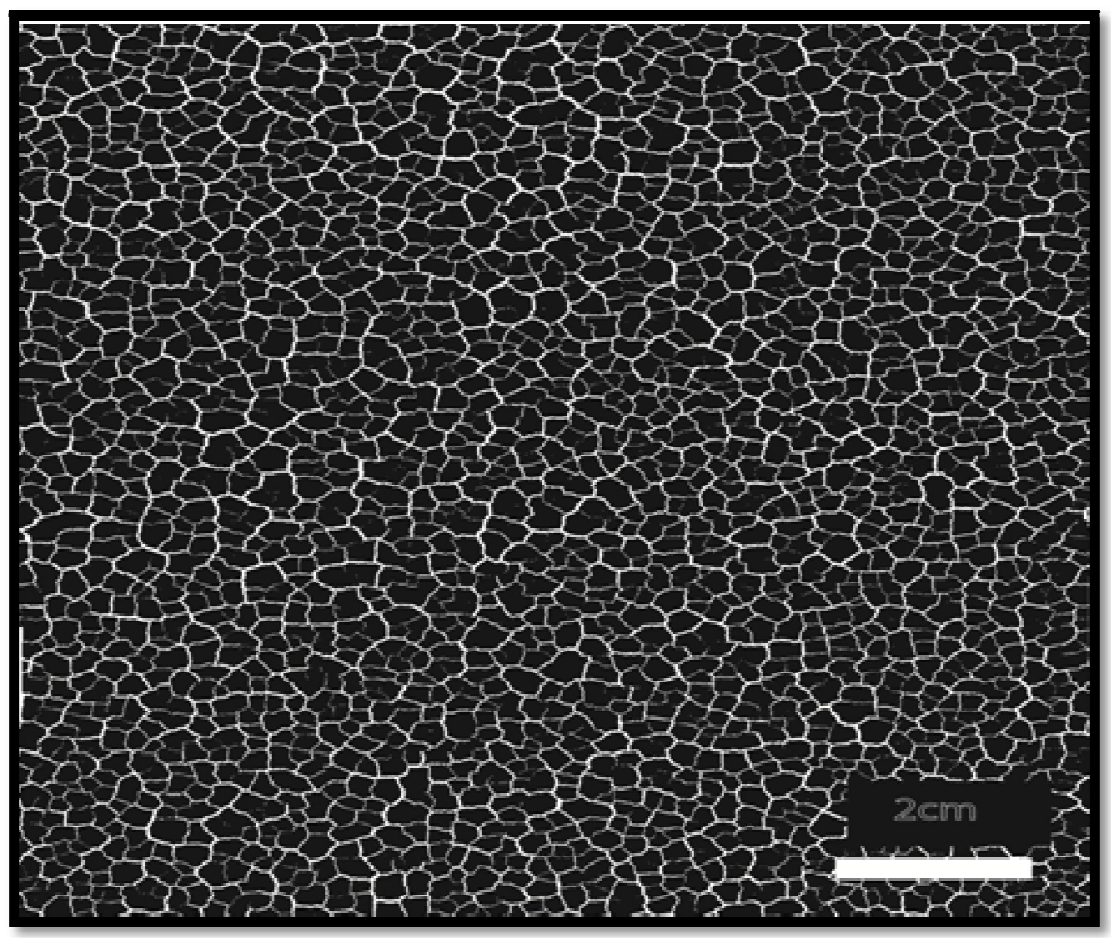

Figure 4: Medium Viscosity Horizontal Raw Starch Experiment of 60g

On stitching the images of the rock analogue with respect to their differences or contrast in colour, brightness, illumination, saturation, etc., two different phases (white and black) are segmented and digitized by RGB threshold in Adobe Photoshop (Peternell \& Kruhl, 2009). In the colour selection menu within the Photoshop, single pixels within specific rock analogue phase are marked and their equivalent RGB colour values noted. The procedure is repeated until a certain colour value which is considered to be the chosen phase is reached. The images are converted to black and white images and the analysed phases represented by black and white phases (figures 3, 4 and 5). The completely stitched images are then analysed in the white and black phases to map the anisotropy of the pattern or fabric using MORFA (Mapping of Rock Forming Anisotropy). In this mapping procedure, gliding of the window is applied to the distribution of the pattern. 


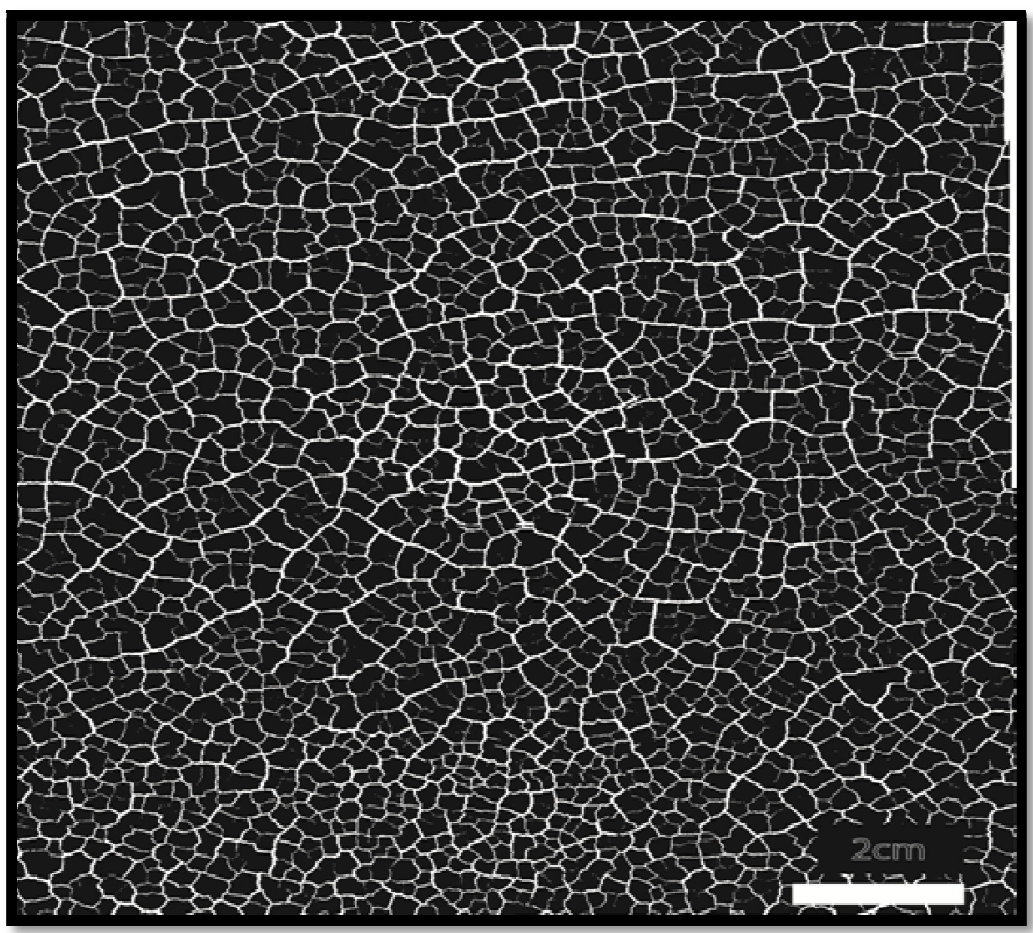

Figure 5: High Viscosity Horizontal Raw Starch Experiment of 80g

\subsection{MORFA Sub-Images}

With MORFA analysis towards the quantification of anisotropic structures, various portions of height and width images were analyzed from the complete pattern (ranging from 1200 to 200 in my case of experiment). That means the box size of each image was defined from the largest image crop possible until the results became unstable. Image for each viscosity was tested and the MORFA software made to run.

I personally adopted the following criteria for selecting good sub-image figures to be analyzed anisotropic with MORFA Analyze:

- The direction of the image should not be widely different from that of the others.

- The correlation coefficient $\left(\mathrm{R}^{2}\right)$ of the ellipse or sphere should be high, at least $75 \%$.

- The correlation coefficient $\left(\mathrm{R}^{2}\right)$ of the corresponding cumulative segment length points should as well be high, at least $75 \%$.

- Good particle or point distribution on the ellipse

It has been observed that the larger the image height and width, the fewer the sub-images to be analyzed and viceversa. Table 2 is the calibration table for the sub-image of figure 6 . The sub-images of the results were plotted using semi-log plot. The log-log plot could have been used since it yielded higher values of the correlation coefficient of the ellipse. However, the cumulative segment length points did not portray any better shapes and curves and as such the points could not define any better gradient. Hence the semi-log plot (which shows both higher values of the correlation coefficient as well as the cumulative segment length points) was employed to plot the sub-images for every measured direction.

\begin{tabular}{|c|c|c|c|c|c|}
\hline Name & Description & $\begin{array}{c}\text { Heigth/ Width } \\
\text { (Px) }\end{array}$ & Orientation $\left({ }^{\circ}\right)$ & $\begin{array}{l}\text { Threshold } \\
\text { Length }\end{array}$ & $\begin{array}{c}\text { Det. Coefficient } \\
(\%)\end{array}$ \\
\hline \multirow{8}{*}{$\begin{array}{c}40 g \\
\text { starch in } \\
\text { water }\end{array}$} & \multirow{8}{*}{$\begin{array}{l}\text { Low viscosity } \\
\text { experiment in } \\
\text { horizontal level }\end{array}$} & $500 * 500$ & 107 & $10-40$ & 84 \\
\hline & & $600 * 600$ & 111 & $10-40$ & 80 \\
\hline & & $700 * 700$ & 120 & $10-40$ & 69 \\
\hline & & $800 * 800$ & 114 & $20-60$ & 75 \\
\hline & & $900 * 900$ & 111 & $20-80$ & 78 \\
\hline & & $1000 * 1000$ & 114 & $20-80$ & 78 \\
\hline & & $1100 * 1100$ & 121 & $20-80$ & 69 \\
\hline & & $1200 * 1200$ & 116 & $20-100$ & 75 \\
\hline
\end{tabular}

Table 2: Low Viscosity Experiment with 40g of Starch Analyzed as Cracks 


\subsubsection{Morfa Sub-Image Diagrams}

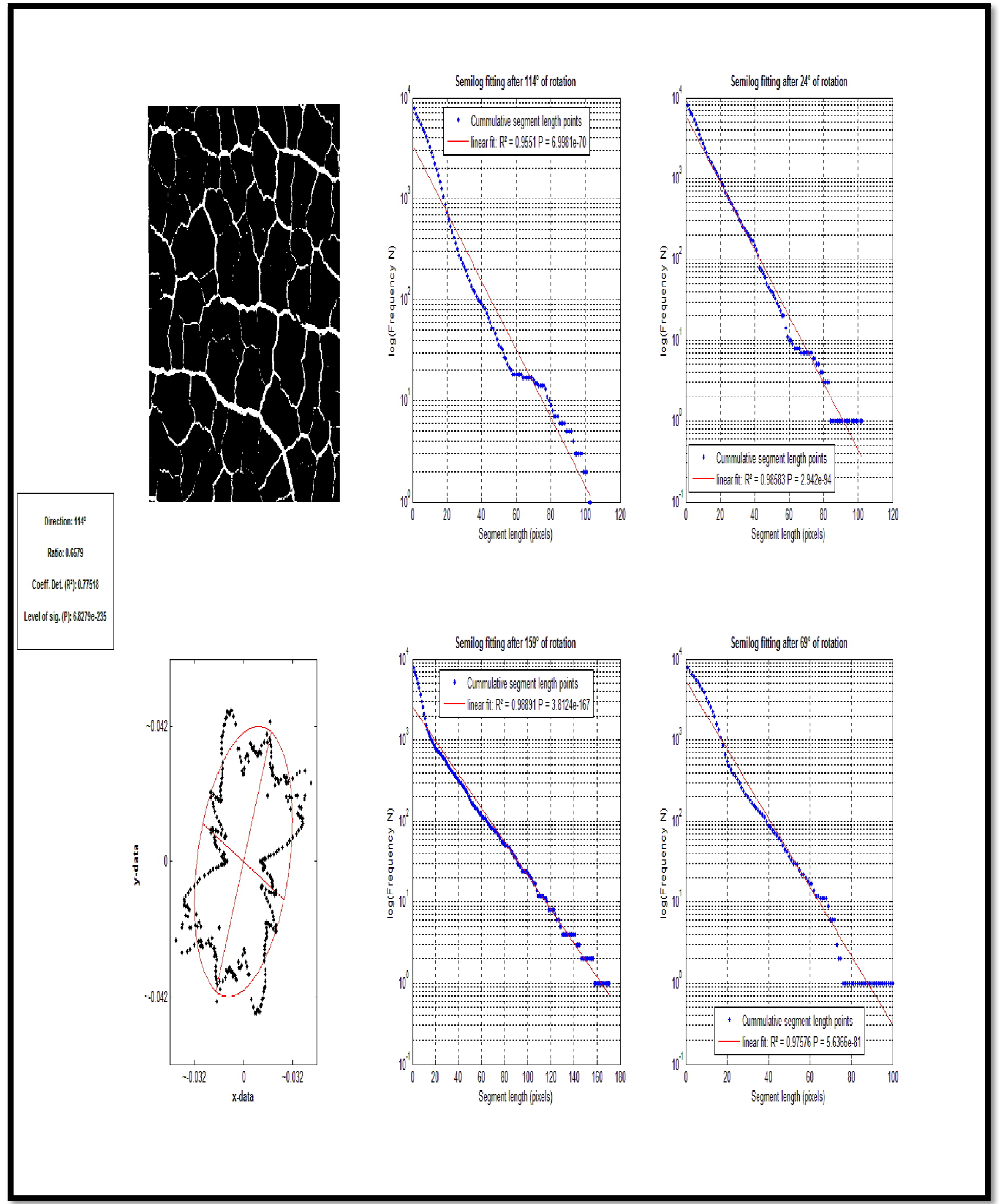

Figure 6: Low Viscosity Horizontal Flow Experiment of 40g Raw Starch Analyzed as Cracks 
The next step is to run the MORFA with the settings for all images and for both black (starch) and white (cracks) phases. I set the following range of overlap for the various heights and widths of the boxes. From $1200 * 1200$ to $1000 * 1000$ heights and widths were set at $95 \%$ overlap, whilst the heights and widths between $900 * 900$ and $800 * 800$ and also from $700 * 700$ down to $200 * 200$ at $90 \%$ and $85 \%$ respectively. A technique to quantify anisotropy of patterns, the measurements ought to be repeated from $0^{\circ}$ to $180^{\circ}$. Fabrics in geo-materials and their related analogues reveal patterns of complex nature. Their sizes, shapes as well as their directions also give information on the physical properties of materials. These fabrics which are mostly anisotropic in direction could be quantified. A quantification of this anisotropy provides information on the anisotropy of the material's physical properties as well as the fabric-forming processes (Kruhl et al., 2004). To analyse and quantify the anisotropy, note must be considered that irregular areas of analysis lead to different length of scan-lines in different directions which can falsify the overall result of quantifying the anisotropy.

The anisotropic directions exhibit the rate of deformation and its flow process. Anisotropy through deformation provides important information about structure forming processes and can be interpreted to be strain. Deformation processes relate to both a flow plane as well as a flow direction (figures $7 \& 8$ ).

\subsubsection{Anisotropy MORFA Images}

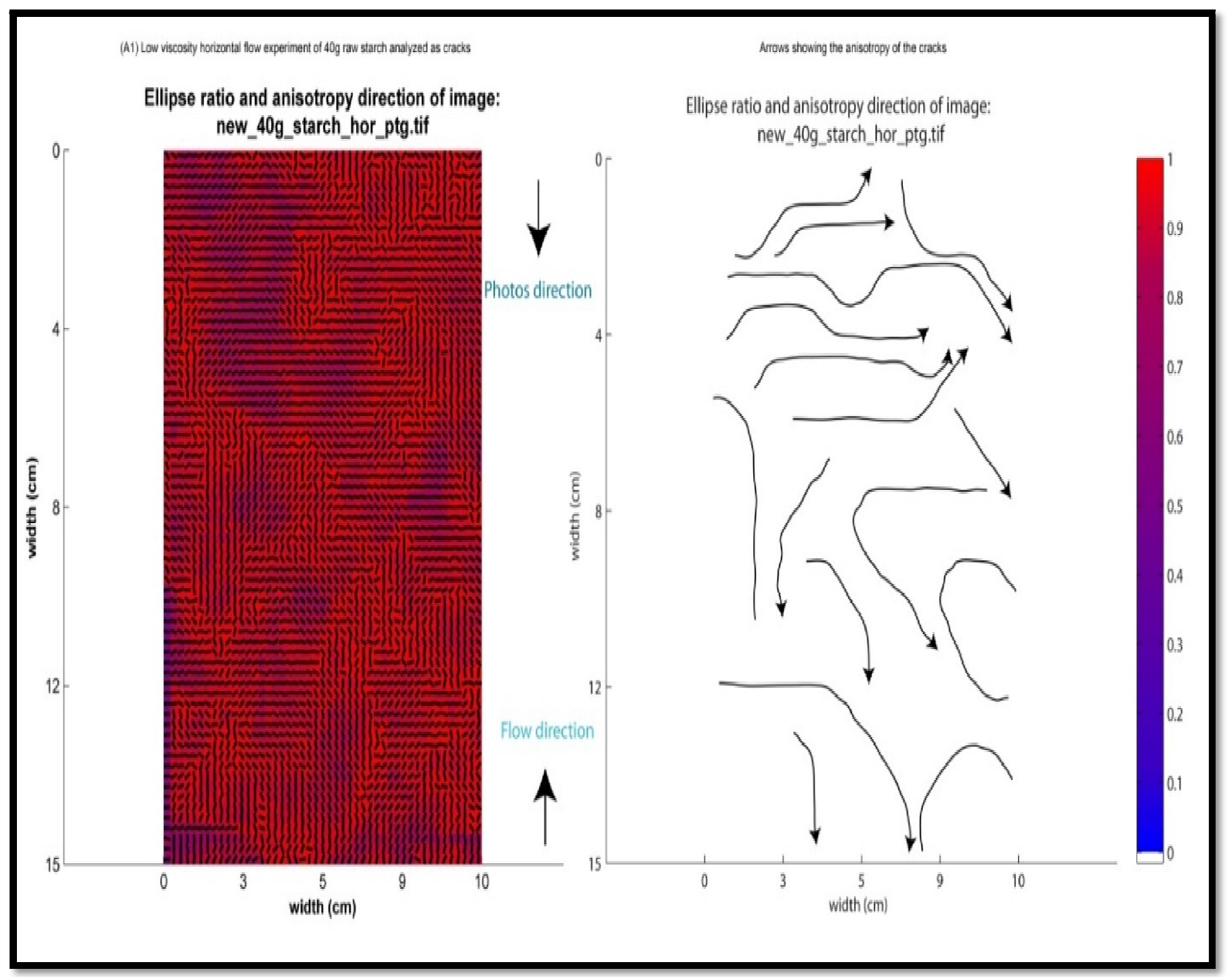

Figure 7: Low Viscosity White Phase MORFA Image of A 40g Raw Starch at Horizontal Flow Analyzed as Cracks with Its Anisotropy (Flow Direction)

To be able to really quantify anisotropy or pattern inhomogeneity, box-counting is defined and applied together with window gliding method (Kruhl, 2012). This leads to quantification of fabrics or patterns of rock analogue, for instance, starch in my case of experiment. This method of fractal analysis was applied to the cracks and starch patterns to establish the fractal nature of the starch and crack or fracture pattern of the rock analogue. 


\subsection{Box-Counting}

The method of box-counting is good at analysing complex patterns of geo-materials as well as their analogues. The box-counting method does not rely on a central symmetry of the pattern (Vignes-Adler et al., 1991). It also analyses pattern of fractures as well as distribution patterns of crystals in magmatic rocks (Peternell \& Kruhl, 2009) and rock analogues. Generally, box-counting is used for mineral and fragment analysis as well as fracture analysis in rocks, minerals and other geomaterials (Kruhl and Nega, 1996). The box-counting method is suitable for giving a quantitative measure of the geometry, the length and the spatial distribution of fracture and fault patterns in any order of magnification and can also be applied to patterns with specific scaling properties (Peitgen et al., 1992, 1998).

Box-counting dimension $\left(D_{b}\right)$ which is a positive real number is defined by the relation $\quad \mathrm{N}(\mathrm{r}) \sim \mathrm{r} \exp \mathrm{D}_{\mathrm{b}} \ldots \ldots$ equation (1) where $\mathrm{D}_{\mathrm{b}}=$ box-counting dimension.

$\mathrm{N}(\mathrm{r})$ is the minimum number of boxes of length $\mathrm{r}$ necessary to cover a 2D pattern. For Euclidean objects, equation (1) defines $\mathrm{D}_{\mathrm{b}}=1$ for a straight line and 2 for a plane (Peternell \& Kruhl, 2009). $1<\mathrm{D}_{\mathrm{b}}<2$.

This method gathers data and uses the gathered data to analyse complex patterns. The analysis is done by breaking the set of data or image into smaller box-shaped sizes. In order to estimate the fractal dimension of the experiment, the Euclidean space containing the image pattern would have to be divided into a grid of boxes of size $\mathrm{r}$. The boxes N(r) which are not empty are counted (Takayasu, 1990). The procedure is repeated with the pattern image at smaller sizes ( $\mathrm{r}$ ) and each time the associated non-empty boxes are counted (Volland \& Kruhl, 2004). Various portions of height and width images were analysed from the complete pattern (ranging from 1200 to 200 Pixels at 100 pixels interval, in this experiment). N(r) is plotted against the reciprocal size $(1 / \mathrm{r})$ of the squares in a double-logarithmic diagram at a scale of $1 \mathrm{~cm}=522$ pixel (figure 9). The required slope defines the fractal dimension (Peitgen et al., 1992, 1998). 


\subsubsection{Box-Count Sub-Images}

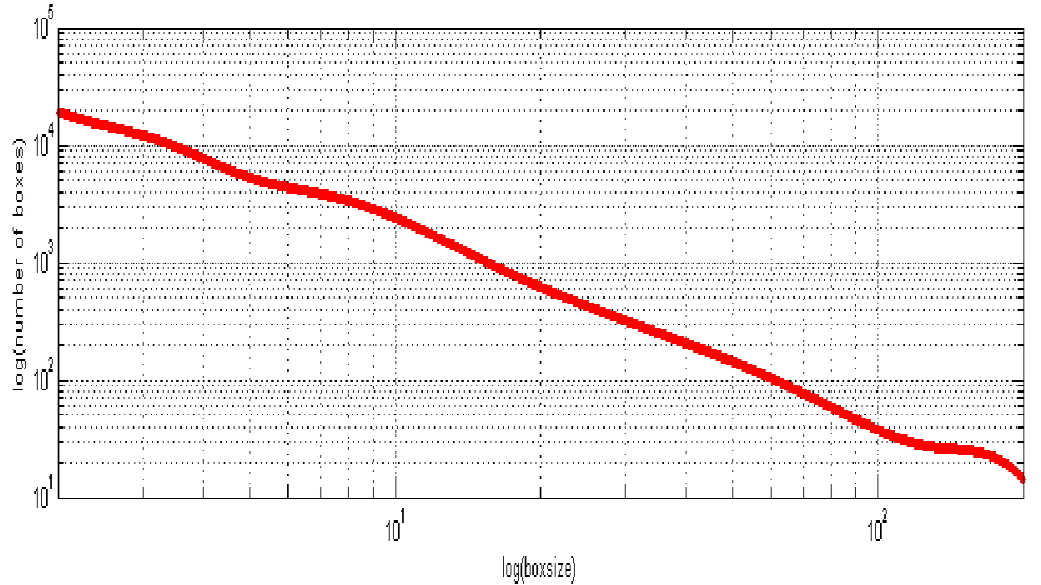

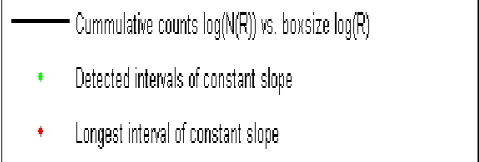
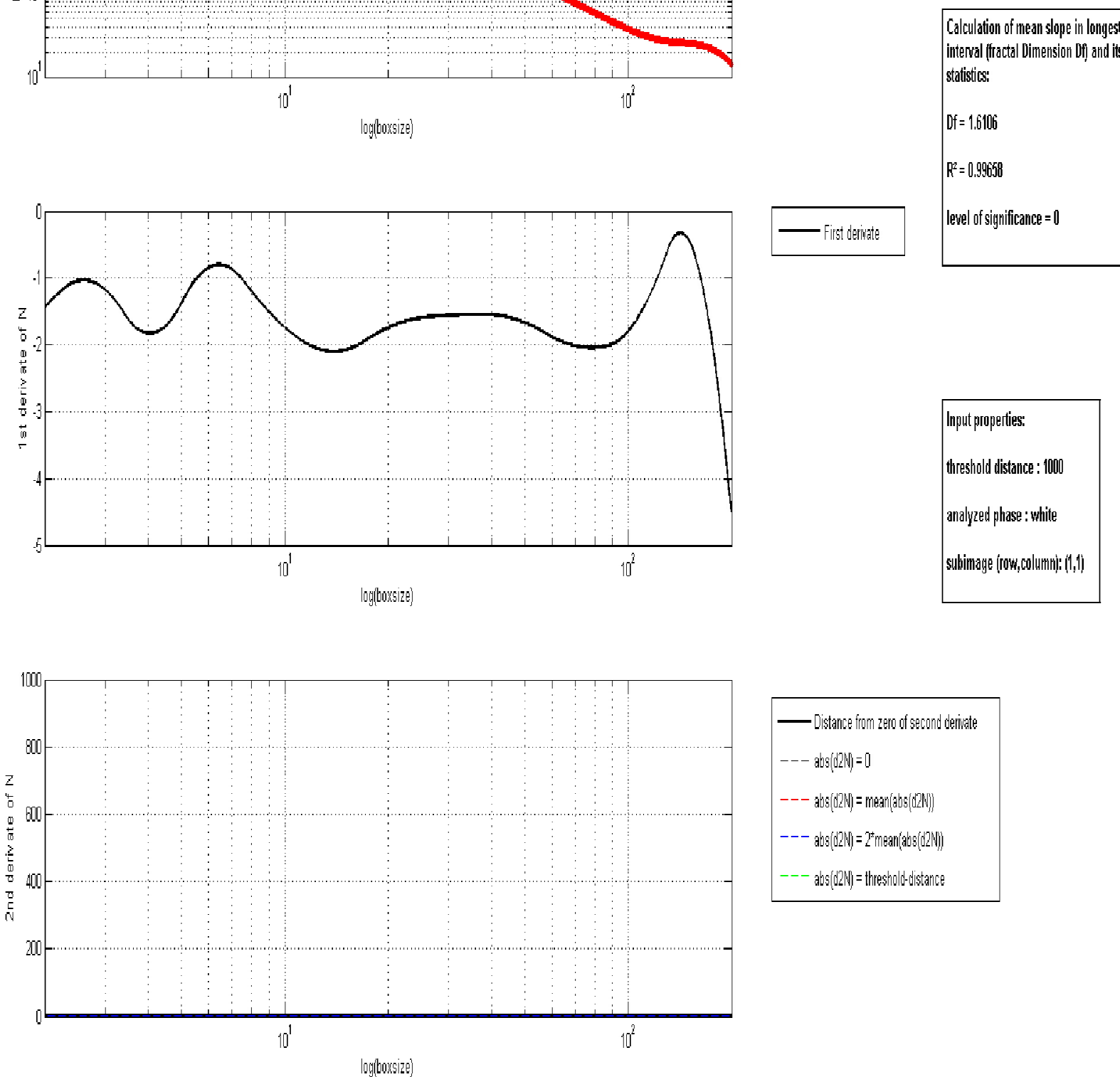

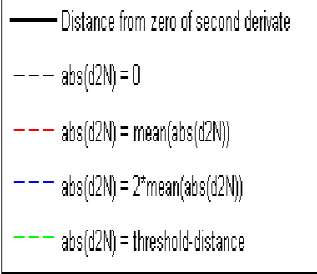

Figure 8: High Viscosity Box-Count Sub-Image of Horizontal Experiment of 80g Starch Analyzed as Cracks 


\begin{tabular}{|c|c|c|c|}
\hline \multirow{4}{*}{ Low Viscosity } & \multirow[b]{2}{*}{ Crack } & $\mathrm{D}_{\mathrm{f}}$ & 1.61 \\
\hline & & $\mathrm{R}^{2}$ & 0.996 \\
\hline & \multirow[b]{2}{*}{ Starch } & $\mathrm{D}_{\mathrm{f}}$ & 1.91 \\
\hline & & $\mathrm{R}^{2}$ & 0.997 \\
\hline \multirow[b]{4}{*}{ Medium Viscosity } & \multirow[b]{2}{*}{ Crack } & $\mathrm{D}_{\mathrm{f}}$ & 1.64 \\
\hline & & $\mathrm{R}^{2}$ & 0.996 \\
\hline & \multirow[b]{2}{*}{ Starch } & $\mathrm{D}_{\mathrm{f}}$ & 1.88 \\
\hline & & $\mathrm{R}^{2}$ & 0.998 \\
\hline \multirow[b]{4}{*}{ High Viscosity } & \multirow[b]{2}{*}{ Crack } & $\mathrm{D}_{\mathrm{f}}$ & 1.61 \\
\hline & & $\mathrm{R}^{2}$ & 0.996 \\
\hline & \multirow[b]{2}{*}{ Starch } & $\mathrm{D}_{\mathrm{f}}$ & 1.90 \\
\hline & & $\mathrm{R}^{2}$ & 0.996 \\
\hline
\end{tabular}

Table 3: Values of Fractal Dimension $\left(\mathrm{D}_{\mathrm{f}}\right)$ and Determination Coefficients $\left(\mathrm{R}^{2}\right)$ of Box-Count

\section{Results and Discussions}

During the low viscosity experiment (figure 3), the rate of flow was very fast. This means, less viscous leads to more flow. This low viscosity starch fluid could flow easily because its molecular makeup results in very little friction when it is in motion. The resistance to flow was very low. At the medium viscosity experiment (figure 4), the flow rate was not fast. It needed time to flow. There was not much resistance to flow and only some water could flow out of it. For the high viscosity flow experiment (figure 5), the rate of flow was very slow. This was because the resistance to flow was very high. It could be stated from here that: "thick fluids have higher viscosity than thin or runny fluids" (Fossen, 2010).

The sizes of the cracks or fractures were differently observed with respect to the different forms of viscosity flow experiments. It was visibly observed that the low viscosity experiment at $40 \mathrm{~g}$ starch produced smaller cracks or fractures (figure 3) whilst the medium viscosity at $60 \mathrm{~g}$ starch (figure 4) and the high viscosity at $80 \mathrm{~g}$ starch experiments (figure 5) produced large and larger fractures respectively. The more viscous the fluid is, the bigger its size of molecules, structure and the cracks. As shown in figures 5, 6 and 7, the entire flow experiment reveals that all the lower viscosities have smaller sizes of molecules and thicknesses of cracks and structure. All the higher viscous fluids reveal larger molecular sizes as well as cracks, whilst the medium viscous fluids have their sizes lying between the lower and higher viscous fluids.

In analysing the right parameters for the MORFA plot to determine the anisotropy, table 2 was considered. Analysing the cracks, it has been observed that analysing a bigger percentage of the total image yields a higher percentage of the determination coefficient $\left(R^{2}\right)$ of both the ellipse and the relationship between the cumulative segment length points and the linear fit as well. In this wise, $1000 * 1000$ Pixels in height and width was considered with the attached parameters to be plotted to determine the anisotropy of the image using MORFA plotting (table 2 \& figure 7). In analysing the starch, higher values of determination coefficient were observed in smaller percentages of height and width with respect to the bigger images. Fabric anisotropy and inhomogeneity are fundamental properties of most natural and artificial crystalline materials and thus fabric or pattern quantification is a necessary prerequisite for studying material properties (Mandelbrot, 1982).

It could be observed from the various plotted images that the more the sub-images leading to the anisotropy plots, the darker the face of the image. Considering most of the cracks (white phase analysis), the chosen height (Pixel) and width (pixel) with the corresponding threshold length and $\mathrm{R}^{2}$ portrayed fewer sub-images compared to most of the black phased images for starch. Higher values of height and width coupled with higher overlap percentage (say $90 \%$ or $95 \%$ ) analyses fewer subimages and thus reveal brighter images. On the other hand, lower values of width and height coupled with lower percentage of overlap (e.g. $85 \%$ ) analyses a lot of sub-images and thus portray darker images. It could be generally observed that the anisotropy of both the cracks and the starch (though diverse directions) start from the inside and sprout out in multiple directions. Almost all the images are directed to both the top and down and sometimes to the other sides. Irrespective of how bright or dark the image is, all the images reveal multi anisotropic nature. However, the darker images, due to their many subimages analysed, portray higher anisotropy.

Of the box-count images (figure 8 and table 3), the slope of the obtained straight line gives us the fractal dimension $\left(D_{\mathrm{f}}\right)$ whilst the accuracy of these straight lines reveals the various correlation values $\left(\mathrm{R}^{2}\right)$. Different ranges of values were detected for both the slope and the accuracy of the straight lines since different phases (white for cracks and black for starch) of analysis were conducted. Straight lines with maximum correlation or fitting or accuracy with better slope were selected. It was generally observed that the correlation coefficients of the black phase in analysing starch ranged between 0.996 and 0.998. In other words, the black phase analysis gave a good linear correlation. However, the low viscosity experiment of starch analysis recorded the highest $\mathrm{R}^{2}$ value indicating the highest accuracy of straight line for the lower viscosity compared to that of the medium and the upper viscosities respectively. The corresponding fractal dimension of this black phase analysis ranged between 1.88 and 1.91 . 
With the white phase analysis, the correlation coefficient attracted a value of 0.996 with fractal dimensions ranging between 1.61 and 1.64. The relatively high box counting dimensions of $1.61-1.64$ and $1.88-1.91$ indicate a non-clustered distribution which argues for a homogeneous drying history (Peternell \& Kruhl, 2009). The box-counting method reveals the fractal behaviour of a pattern. For instance, a pattern in 2D the method leads to a fractal dimension between 1 and 2 (Peternell $\&$ Kruhl, 2009). Since the fractal dimension of this experiment lies between 1 and 2, it falls between a line and a plane. The aforementioned measurements or values show a narrow range of fractal dimensions and thus do not indicate multi-fractal behaviour and the structures are self-similar and also the process is a single pattern-forming process.

The correlation coefficients of the starch analyses gave good linear fitting and were highly recorded at $0.997-0.998$. These yielded corresponding higher values of fractal dimension ranging from 1.88 to 1.91. The white phase analysis (cracks) showed correlation coefficient of 0.996 which is highly considered. The starch analyses which portrayed correlation coefficients of $99.8 \%$ show very good results for natural fractals and the structures portray self-similarity in the measurements. The structures in the white analyses revealed good pictured contours and windows. However, in the black phase analyses, all the structures revealed some amount of contours.

\section{Significance to Tectonic Activities}

Considering for instance, the Gutenberg-Richter relationship, $\mathrm{D}_{\mathrm{f}}=1.8$ for distributed seismicity on earthquake. Also, the frequency-magnitude distribution of seismicity in Southern California from 1932 to 1994 has $D_{\mathrm{f}}$ of 1.846 (Sieh et al., 1989). This helps in acquiring information on radiocarbon dating of faults and folds. Barton (1995) performed systematic studies on exposed joint and fault-trace patterns on Yucca Mountain in Nevada. The fractal statistics were good correlated and the fractal dimensions ranged from 1.4 to 1.7. Measurements in geology and geophysics that yield power law spectra have their fractal behaviour lying between $1<\mathrm{D}<1.6$. Takayasu, 1985 proposed the deterministic model of fracture, and ascertained the fractal dimension to be about 1.65 for the fracture pattern. The Smithsonian / NASA Astrophysics Data System have indicated the fractal dimension $D_{\mathrm{f}}$ is about 1.5-1.6 at the central part of the Japan Arc, and decreases with distance from the centre. T. Hirata, 1989 gave the fractal dimension of the fracture geometry of rocks as 1.6.

\section{Conclusion}

Considering the low, medium and high viscous fluids in my experiment, the less viscous fluids lead to more flow and vice-versa. The molecular make-up plays an active role in the flow capacity. The lower the viscosity and density of the fluid, the lower the friction and resistance to flow resulting in easy flow and vice-versa. Also, the rate of viscosity and density of the fluid correspond to the size of fractures or cracks produced. Lower viscosity and lower density produce smaller cracks and viceversa. The ease with which fluids flow is described in terms of their viscosity and viscous deformation implies dependence of stress on strain rate: higher stress means faster flow or more rapid strain accumulation (Fossen, 2010). At low viscosity, there is higher stress and as such the flow is rapid which in turn accumulates strain. The type of flow in this wise is turbulent. However, at higher viscosity, lower stress is built up corresponding to lower strain accumulation. This results in slower movement of the starch and laminar flow is pronounced. The presence of melt affects the rheology of the Earth's crust, regional deformation and crustal rheological instabilities. Over geologic time, rocks in the middle and lower crust can be considered as fluids with very high viscosity (Fossen, 2010). This is due to the fact that there is very small strain in the upper crust and higher in the lower and middle crust. Since lower stress and strain correspond to higher viscosity, the upper crust will have higher viscosity. The weak and viscous nature of salt enables much wider areas to be deformed (Fossen, 2010).

In determining the anisotropy of the starch and the cracks with MORFA software, almost all the patterns exhibit anisotropic flow process. Anisotropy thus exhibits the rate of deformation and the strain of the ellipse and the deformation process. It could also be concluded that the size of the sub-images determines the brightness or colour of the image. Thus the more the sub-images leading to the anisotropy plots, the darker the surface of the image and vice-versa.

The high coefficient of determination for both the cracks and the starch analyses becomes the basis of determination of the quality of linear regression (Peternell \& Kruhl, 2009).

A minimum coefficient of determination of 0.996 is used with respect to this experiment. High box-count values of 1.88-1.91 (black phase) and 1.61-1.64 (white phase) leads to complex phase distribution patterns. Box counting has established itself as a suitable method for quantifying the complex distribution patterns of minerals, rock structures and their analogues as well. The high values of the box counting dimensions of starch with its various mixtures at both the white and black phases can be said to be for a homogeneous crystallization process leading to non-clustered and complex phase distribution patterns.

From my experiment, both the box-count and map-count values of $\mathrm{D}_{\mathrm{f}}$ for the starch portrayed averagely 1.9 and corresponds almost to this data of the frequency-magnitude distribution of seismicity in Southern California from 1932 to 1994 by Sieh et al., 1989 of $D_{\mathrm{f}}$ of 1.85; and also that of the Gutenberg-Richter relationship, $\mathrm{D}_{\mathrm{f}}=1.8$ for distributed seismicity on earthquake. $\mathrm{D}_{\mathrm{f}}$ for the cracks or fractures have average value of 1.62 which corresponds to the $\mathrm{D}_{\mathrm{f}}$ values of Takayasu, NASA, Hirata, Barton and that of the general $\mathrm{D}_{\mathrm{f}}$ data for geophysics. These values on earthquakes, joints and faults give revelation of the partings of weaknesses through which the magma flows to produce magmatic rocks. During intensive earthquake the crust undergoes deformation in the form of faulting or jointing. These places of weaknesses act as channel ways for the transmission of heat from the lower layers to the upper layers of the earth crust. This influx of heat from the lower layers causes 
considerable melting in the upper part of the crust. The rock material which is subsequently melted forms magmatic rocks. Magmatic rocks are formed from the cooling and subsequent solidification of hot molten rock material called magma. Magma migrates along these parting planes or planes of weakness such as fault or joint to the surface of the earth.

For Euclidean objects, $\mathrm{D}_{\mathrm{f}}=1$ for a straight line and 2 for a plane (Peternell \& Kruhl, 2009). $1<\mathrm{D}_{\mathrm{f}}<2$. Both the starch and cracks of my experiments could be quantified to lie between 1 and 2. This means both the starch and cracks quantified values can be found between a line and a plane.

Geological structures could be grouped differently on different scales (pattern scaling), in different areas (pattern inhomogeneity) and in different directions (pattern anisotropy) (Kruhl, 2012).

In conclusion, a rock analogue can be quantified to achieve the desired results the same way it is done with the original rock.

\section{Acknowledgement}

I am grateful to the lecturers of the Department of Geological Engineering for their unflinching support to bring this paper to fruition. Thanks also to my Teaching Assistants, Mr. Isaac Kotei and Camilius Amevorku for their diverse support on this paper. To all and sundry, I say thank you.

\section{References}

i. Barton, C.C., 1995: Fractal analysis of scaling and spatial clustering of fractures. In: Barton, C.C., La Pointe, P.R. (Eds.), Fractals in the Earth Sciences. Plenum Press, New York, pp. 141-178.

ii. Fossen, H., 2010: Structural Geology. Cambridge University Press. Gillespie et. al., 1993: The Nature and Tectonic significance of Fault Zone Weakening.

iii. Grady and Kipp, 1987: Dynamic Rock Fragmentation.

iv. Hirata, T., 1989. Fractal dimension of fault systems in Japan; Fractal structure in rock fracture geometry at various scales. Pure and Applied Geophysics 131 (1-2), 157-170.

v. Kruhl, J.H., 2012. Fractal-geometry techniques in the quantification of complex rock structures: A special view on scaling regimes, inhomogeneity and anisotropy. Journal of Structural Geology xxx (2012) 1-20.

vi. Kruhl, J.H., \& Nega, M., 1996. The fractal shape of sutured quartz grain boundaries: application as a geothermometer. Geologische Rundschau 85, 38-43.

vii. Mandelbrot, B.B., 1967. How long is the coast of Britain? Statistical self-similarity and fractional dimension. Science, $156,636-638$

viii. Mandelbrot, B.B., 1977: Fractals - Form, Chance and Dimension. Freeman. San Francisco. Pp 365

ix. Mandelbrot, B.B., 1982. The fractal geometry of nature. Freeman \& Co. New York 468.

x. Peitgen et. al., 1992, 1998. Chaos and Fractals: New Frontiers of Science.

xi. Peternell, M., \& Kruhl J.H, 2009. Automation of pattern recognition and fractal-geometry-based pattern quantification, exemplified by mineral-phase distribution patterns in igneous rocks. Computers \& Geosciences, 35, 1415-1426.

xii. Sieh et. al. 1989. A more precise chronology of earthquakes produced by the San Andreas Fault in southern California. Journal of geophysical research, 94 .

xiii. Takayasu, H., 1990. Fractals in the Physical Sciences. Manchester University Press, Manchester, pp. 170.

xiv. Vignes-Adler et. al., 1991. Fractal analysis of fracturing in two African regions from satellite imagery to ground scale. Tectonophysics, 196, 69 - 86.

xv. Volland, S., \& Kruhl, J.H., 2004. Anisotropy quantification: the application of fractal geometry methods on tectonic fracture patterns of a Hercynian fault zone in NW-Sardinia. Journal of Structural Geology, 26, 1489-1500. 